\title{
High SIRT1 expression is a negative prognosticator in pancreatic ductal adenocarcinoma
}

\author{
Albrecht Stenzinger ${ }^{1 *}$, Volker Endris ${ }^{1}$, Frederick Klauschen ${ }^{2}$, Bruno Sinn ${ }^{2}$, Katja Lorenz $^{1}$, Arne Warth ${ }^{1}$, \\ Benjamin Goeppert', Volker Ehemann', Alexander Muckenhuber ${ }^{1}$, Carsten Kamphues ${ }^{3}$, Marcus Bahra ${ }^{3}$, \\ Peter Neuhaus ${ }^{3}$ and Wilko Weichert ${ }^{1}$
}

\begin{abstract}
Background: Several lines of evidence indicate that Sirt1, a class III histone deacetylase (HDAC) is implicated in the initiation and progression of malignancies and thus gained attraction as druggable target. Since data on the role of Sirt1 in pancreatic ductal adenocarcinoma (PDAC) are sparse, we investigated the expression profile and prognostic significance of Sirt1 in vivo as well as cellular effects of Sirt1 inhibition in vitro.

Methods: Sirt1 expression was analyzed by immunohistochemistry in a large cohort of PDACs and correlated with clinicopathological and survival data. Furthermore, we investigated the impact of overexpression and small molecule inhibition on Sirt1 in pancreatic cancer cell culture models including combinatorial treatment with chemotherapy and EGFR-inhibition. Cellular events were measured quantitatively in real time and corroborated by conventional readouts including FACS analysis and MTT assays.

Results: We detected nuclear Sirt1 expression in 36 (27.9\%) of 129 PDACs. SIRT1 expression was significantly higher in poorly differentiated carcinomas. Strong SIRT1 expression was a significant predictor of poor survival both in univariate $(p=0.002)$ and multivariate (HR 1.65, $p=0.045)$ analysis. Accordingly, overexpression of Sirt1 led to increased cell viability, while small molecule inhibition led to a growth arrest in pancreatic cancer cells and impaired cell survival. This effect was even more pronounced in combinatorial regimens with gefitinib, but not in combination with gemcitabine.
\end{abstract}

Conclusions: Sirt1 is an independent prognosticator in PDACs and plays an important role in pancreatic cancer cell growth, which can be levered out by small molecule inhibition. Our data warrant further studies on SIRT1 as a novel chemotherapeutic target in PDAC.

Keywords: Pancreatic cancer, HDAC, Sirt1, Biomarker, Pancreatic ductal adenocarcinoma

\section{Background}

Pancreatic ductal adenocarcinoma (PDAC) is the fourth leading cause of cancer related deaths in the United States. While substantial progress has been made in the understanding of pancreatic cancer biology [1], therapeutic concepts still provide only modest benefit [2]. The overall 5 -year survival rate is approximately $5 \%$ [3]. Surgical resection is the only efficient and potentially curative

\footnotetext{
* Correspondence: albrecht.stenzinger@med.uni-heidelberg.de 1 Institute of Pathology, and National Center for Tumor Diseases (NCT), University Hospital Heidelberg, Heidelberg, Germany

Full list of author information is available at the end of the article
}

treatment option with 5-year survival rates of around $20 \%$ in patients with resectable tumors, but can only be applied in approximately $15-20 \%$ of the cases emphasizing the urgent need for early detection strategies [4].

The main prognosticators for surgically resectable PDACs are location, tumor size, resection margin, nodal status, and histological grade. Although these risk factors have been proven to be clinically useful, their ability to reliably predict outcome is limited and mainly reflects tumor distribution rather than tumor biology [4].

Hence, numerous studies have been conducted to identify novel biomarkers that aid outcome prediction and to

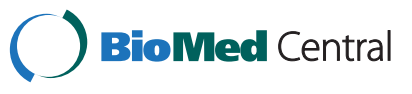


unravel molecular mechanisms that drive tumor development [5].

Sirt1 (homolog of yeast silent information regulator, Sir2), an isoform of enzymes of the silent information regulatory family (sirtuins), is an evolutionary conserved NAD dependent histone/protein deacetylase (class III HDAC) that mediates epigenetic silencing by modification of lysine residues of histones and deacetylation of numerous non-histone substrates. One of the first substrates identified was p53, whose deacetylation was reported to repress p53-dependent apoptosis in response to cellular stress and DNA damage [6,7]. Meanwhile, many other targets have been identified, including Ku70 [8], PTEN [9], p73 [10], RelA/p65 [11], FOX01, FOX03a, and FOX04 [12], NICD [13], hypoxia-inducible factors HIF-1 $\alpha,-2 \alpha$ $[14,15], \beta$-catenin [16], XPA [17], SMAD7 [18], and cortactin [19]. Deacetylation of these targets regulates cell survival, proliferation, and angiogenesis. Overexpression of sirtuins was initially reported to increase lifespan in budding yeast, Caenorhabditis elegans, and Drosophila melanogaster [20-22] but for the latter two the findings were challenged by a recent study of Burnett and colleagues [23].

The functional role of Sirt1 in cancer is equivocal and suggested to be context dependent [24]. Although there are convincing studies that argue for a tumour suppressive role of Sirt1, recent data provide functional evidence that Sirt1 has oncogenic properties in neuroblastomas by facilitating n-myc stabilization [25]. Serrano [26] reported that transgenic Sirt mice crossed with PTEN-null mice were observed to develop thyroid and prostate cancer further arguing for a tumor promoting function of Sirt1.

While several studies found deregulation of Sirt1 in various tumor entitites including ovary, prostate, gastric, colon, hepatocellular carcinoma as well as melanoma and glioblastoma [27], comprehensive in vivo data in pancreatic cancer is still missing. Reports that explore Sirt1 function in pancreatic cancer are sparse [28].

Hence, we set out to comprehensively investigate Sirt1 expression in a large series of PDACs, its relationship to survival and to assess the functional relevance in cell culture models.

\section{Methods}

\section{Patients and samples}

Tissue samples from 129 patients who underwent partial pancreaticoduodenectomy for primary pancreatic ductal adenocarcinoma between 1991 and 2000 were retrieved from the database of the Pathology Department of the Charité University Hospital. The study was approved by the Charité University Ethics Committee (No. EA1/06/ 2004).

Median age of patients with pancreatic cancer was 65 years (range $35-80$ years). Follow-up data regarding overall survival were available for 113 patients. Within the follow up time, 89 patients $(78.8 \%)$ died after a mean follow up time of 22.1 months. Mean follow-up time of patients still alive at the endpoint of analysis was 54.0 months. Cases were staged according to "TNM Classification of Malignant Tumours. 7th edition" [29] and were graded as recommended by the WHO [30].

\section{Tissue microarray construction}

Of all PDACs $3-\mu \mathrm{m}$ sections were cut and stained with H\&E. Three representative areas from the tumor center and invasive margins were marked by a board certified pathologist (W.W.). For each case three tissue cores (1.5$\mathrm{mm}$ diameter) from the selected representative tumor areas were punched out of the sample tissue blocks and embedded into a new paraffin array block using a tissue microarrayer (Beecher Instruments, Woodland, CA).

\section{Immunohistochemistry}

For immunohistochemical detection of Sirt1 on tissue samples, a monoclonal rabbit antibody (dil.: 1:100, clone E104, Cat\# 1104-1; Epitomics, Burlingame, CA, USA) was used.

After heat-induced antigen retrieval, slides were incubated with the primary antibody at 4 degree Celsius overnight. Bound antibody was detected by a streptavidin-biotin system (BioGenex, San Ramon, CA, USA). For colour development, a Fast Red system (Sigma, Deisenhofen, Germany) was used. Omission of the primary antibody served as negative control. The slides were cover slipped after counterstaining.

Nuclear staining of Sirt1 was scored by applying a semiquantitative immunoreactivity scoring (IRS) system, as described previously. Briefly, the intensity of staining and percentage of cells stained were evaluated separately. The IRS for each individual case ranging from 0 to 12 was calculated by multiplication of the intensity and frequency scores. Cases exhibiting an IRS from 0-6 were combined in one group ('Sirt1 low'), cases with an IRS of $>6$ were combined in a 'Sirt1 high' group. Staining of tissue slides was evaluated by experienced pathologists (WW and AS) blinded towards patient characteristics and outcome.

\section{Cell culture}

The human pancreatic cancer cell lines PANC-1 (\#CRL1469) and MiaPaCa-2 (\#CRL-1420) were obtained from the American Type Culture Collection (ATCC, Rockville, MD, USA) and cultured in Dulbecco's modified Eagle's medium supplemented with $10 \%$ fetal bovine serum and $\mathrm{P} / \mathrm{S}$. For the MIA-PaCa- 2 cells, additionally $2.5 \%$ horse serum and $5 \mathrm{ml} \mathrm{NaHCO} 3(0.75 \mathrm{mg} / \mathrm{ml}$ final concentration $)$ were used. These two cell lines were chosen, since PANC-1 is a prototypical Gemcitabine resistant cell line, while Mia-PaCa-2 is known to retain some Gemcitabine sensitivity. 


\section{Reagents}

Cambinol (Cat\#566323) was purchased from Merck (Darmstadt, Germany), Gefitinib (Cat\#PKI-GFTB2-200) was obtained from Biaffin (Kassel, Germany) and Nicotinamide from Sigma (Taufkirchen Germany).

\section{Plasmids, siRNA and transfections}

The SIRT1/2 and GFP control expression constructs were obtained from Addgene. For SIRT1, expression of the FLAG-tagged SIRT1 open reading frame was under the control of an SV40 promotor, allowing physiological levels of SIRT1 expression in cells not harbouring the Large-T antigen (pECE-FLAG-SIRT1, constructed by Michael Greenberg [31]). GFP (Addgene plasmid 13031, constructed by Doug Golenbock) was cloned in a pcDNA3 vector, allowing high protein expression controlled by $\mathrm{CMV}$ promotor. Predesigned siRNAs for Sirt1 were purchased from Dhamarcon (ON-TARGETplus SMARTpool, human Sirt1, Cat\# L-003540-00-0010). The target sequence is as follows: GCGAUUGGGUACCGAGAUA. A non-target scambled siRNA was used as negative control (all stars negative control siRNA; Cat\#1027281, Qiagen, Hilden Germany). After $72 \mathrm{~h}$, the efficacy of transfection was checked by immunoblotting.

All transfections were performed using oligofectamine (dilution: 1:200; Invitrogen, Karlsruhe, Germany) according to the manufacturers' protocol.

\section{MTT assay}

Cell viability was measured 72 hrs after pSirt1 transfection by the MTT (3-[4,5-dimethylthylthiazol-2-yl]-2,5diphenyltetrazolium bromide; Sigma, Munich, Germany) assay according to the manufacturer's instructions. Briefly, $20 \mu \mathrm{l}$ of $5 \%$ MTT solution in PBS was added to each well. After 4-6 h of incubation at $37^{\circ} \mathrm{C}$, the active dehydrogenase in viable mitochondria reduced the tetrazolium ring of MTT to form a blue-colored precipitate, which was then dissolved in $150 \mu \mathrm{l} 50 \%$ dimethyl sulfoxide / 50\% Ethanol and quantified spectro-photometrically at $570 \mathrm{~nm}$.

\section{Real time analysis}

The PANC-1 and MiaPaCA-2 cell lines were seeded in designated 96 well E-plates (Roche, Penzberg, Germany). Impedance-based real time detection of cellular proliferation was conducted using the xCELLigence system Real-Time Cell Analyzer RTCA-SP (Roche Diagnostics, Penzberg, Germany). The impedance readout as recorded by the $\mathrm{xCELLigence} \mathrm{system} \mathrm{is} \mathrm{converted} \mathrm{into} \mathrm{arbitrary} \mathrm{cell} \mathrm{index}$ (CI)-values corresponding to each well. The CI value is defined as relative change in measured electrical impedance to represent cell status, and is directly proportional to quantity, size, and attachment forces of the cell. Recording of CI and subsequent normalization of the cell index (normalized cell index, NCI) was performed using the RTCA Software 1.2 (Roche).

The NCI is calculated using the equation: $\mathrm{NCI}=\mathrm{CI}$ at a given time point divided by the $\mathrm{CI}$ at the normalization time point. Hence, the NCI equals 1 at the normalization time point. Background impedance caused by the media was determined in each well before seeding the cells and subtracted automatically by the RTCA software following the equation: $\mathrm{CI}=(\mathrm{Ri}-\mathrm{R} 0) / 15$ with $\mathrm{Ri}$ as the impedance at any given time point and $\mathrm{R} 0$ as the background resistance.

\section{FACS analysis}

The effect of Cambinol and Gefitinib on the cell-cycle profile of pancreatic cancer cells was assessed by flow cytometry. PANC-1 and MiaPaCa-2 were exposed to various concentrations of Cambinol or Gefitinib or combinations thereof for $14 \mathrm{hrs}$ and $72 \mathrm{hrs}$ and the cell cycle profiles were determined by flow cytometry as described previously [32]. Briefly, the cells were harvested with versene, treated with a citric acid buffer (2.1\% citric acid and $0.5 \%$ Tween 20 in $\mathrm{dH}_{2} \mathrm{O}$ ), and stained using a phosphate buffer

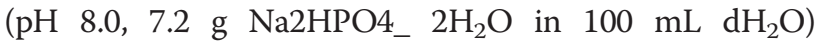
containing DAPI. DNA-histograms were obtained by flow cytometry (PAS II, Partec; Muenster, Germany) and the Multicycle program (Phoenix Flow Systems, San Diego, USA) was used for histogram analysis. Each measurement was done in triplicate.

\section{Immunoblotting}

Treated PANC-1 and MiaPaCa-2 cells were lysed in cell lysis buffer (\#9803, New England.

Biolabs, Frankfurt, Germany) containing $20 \mathrm{mM}$ Tris$\mathrm{HCl}$ (pH 7.5), $150 \mathrm{mM} \mathrm{NaCl}, 1 \mathrm{mM} \mathrm{Na} 2$ EDTA, $1 \mathrm{mM}$ EGTA, 1\% Triton, $2.5 \mathrm{mM}$ sodium pyrophosphate, $1 \mathrm{mM}$ beta-glycerophosphate, $1 \mathrm{mM} \mathrm{Na} \mathrm{VO}_{4}, 1 \mu \mathrm{g} / \mathrm{ml}$ leupeptin as well as Protease inhibitor Mix G (\#39101.01; Serva Electrophoresis, Heidelberg, Germany). Prepared protein lysates $(30 \mu \mathrm{g})$ were denaturated at $95{ }^{\circ} \mathrm{C}$, separated in sodium dodecyl sulfate polyacrylamide (SDS)-polyacrylamide gels (10\%) by electrophoresis and electro-transferred to a polyvinylidene difluoride (PVDF) membrane. After transfer, samples were blocked with 5\% MP-PBST for $1 \mathrm{~h}$ and probed with antibodies against Sirt1 (dil.: 1:5000, clone E104, Cat\# 1104-1; Epitomics), cleaved PARP (dil.: 1:300, Cat\# 9541, clone Asp214 ; Cell Signaling), pospho-H2AX pSer139 (dil: 1:000, Cat\# 05-636, clone JBW 301; Millipore) and beta-Actin (dil: 1:10000, Cat\#A5441, clone AC-15; Sigma) diluted in 5 MP-PBST ( $5 \%$ milk powder, Phosphatebuffered saline/Tween) and incubated at $4{ }^{\circ} \mathrm{C}$ overnight. The appropriate secondary antibody was applied [1:20000; horseradish peroxidase anti-mouse and horseradish peroxidase anti-rabbit] at room temperature for $1 \mathrm{hr}$. Visualization was performed by enhanced chemiluminescence (Amersham Bioscience, Freiburg, Germany). 
Western blots signals were quantified using the ImageJ 1.32 software (National Institutes of Health, Bethesda, MD) after scanning of the films.

\section{Statistical analysis}

For correlation analysis of Sirt1 expression with clinicpathological parameters, the Fisher's exact test or $\chi^{2}$ test for trends was applied. For univariate analysis we used the Kaplan-Meier method and a Log-rank test to probe for significance. For multivariate survival analysis the Cox proportional hazard method was used. Variables found in univariate analysis to be significantly related to survival were included in the Cox models. For statistical analysis of cell cycle and MTT data, a two-tailed t-test was applied. For all statistical tests and methods, $\mathrm{p}$-values of $<0.05$ were considered statistically significant. Statistical analyses were carried out with SPSS 15.0 and Graph Pad Prism 4.

\section{Results}

\section{Patients' and tumor characeristics}

The patients' demographics are listed in Table 1. The mean follow-up time was 22.1 months. During the study period, 89 patients died. The median survival was 13.4 months and the median time to death was 10.3 months (range: 1.2 to 41.93 months). 65 patients were below the age of 65 and 64 patients above the age of 65 (median $65 \mathrm{yrs}$ ). 118 PDAC were located in the head of the pancreas and 11 in the pancreatic corpus or tail.

\section{Sirt1 expression in PDACs}

The specificity of the antibody used for immunohistochemistry was corroborated by siRNA-mediated knockdown of Sirt 1 in MiaPaCa-2 and PANC-1 cells and subsequent immunoblotting with the Sirt1 antibody. The knock-down led to complete abrogation of the immunosignal as shown in Figure 1.

As exemplified in Figure 2, we observed a nuclear localization of Sirt1 in PDAC with a low expression (Figures $2 \mathrm{~A}$ and $\mathrm{B}$ ) in $72.1 \%$ and a high expression (Figures 2C and D) in $27.9 \%$ of the cases, respectively. Sirt1 was expressed by tumor cells with varying degrees of nuclear atypia, forming either neoplastic duct like structures, solid masses or single cell infiltrates within desmoplastic stroma.

When analyzed with regard to the morphological features and tumor extent, the expression of Sirt1 was significantly correlated to poor histological differentiation $(\mathrm{p}=0.001)$. There was no statistical difference in Sirt1 expression between early stage and advanced stage tumors (WHO stage and TNM stage, Table 1).

\section{Univariate survival analysis}

By univariate survival analysis (Table 2), patients' outcome was correlated with both tumor TNM and WHO stage
Table 1 Clinico-pathological characteristics of the PDAC study cohort: correlation with Sirt 1 expression

\begin{tabular}{|c|c|c|c|c|}
\hline Characteristics & All cases & SIRT1 low & SIRT1 high & $\mathrm{p}$-value \\
\hline \multicolumn{5}{|l|}{ All cases } \\
\hline & 129 & $93(72.1 \%)$ & $36(27.9 \%)$ & \\
\hline Age & & & & 0.401 \\
\hline$\leq 65$ years & $65(50.4 \%)$ & $48(73.8 \%)$ & 17 (26.2\%) & \\
\hline$>65$ years & $64(49.6 \%)$ & $48(70.3 \%)$ & 17 (29.7\%) & \\
\hline WHO stage & & & & 0.871 \\
\hline I & $12(9.3 \%)$ & $9(75 \%)$ & $3(25 \%)$ & \\
\hline$\| \mathrm{A}$ & 17 (13.2\%) & $13(76.5 \%)$ & $4(23.5 \%)$ & \\
\hline$\| \mathrm{B}$ & 91 (70.5\%) & $66(72.5 \%)$ & $25(27.5 \%)$ & \\
\hline III & $5(3.9 \%)$ & $3(60.0 \%)$ & $2(40.0 \%)$ & \\
\hline IV & $4(3.1 \%)$ & $2(50.0 \%)$ & $2(50.0 \%)$ & \\
\hline Tumour stage & & & & 0.793 \\
\hline $\mathrm{T} 1$ & / & / & / & \\
\hline $\mathrm{T} 2$ & $24(18.6 \%)$ & $18(75.0 \%)$ & $6(25.0 \%)$ & \\
\hline T3 & $100(77.5 \%)$ & $72(72.0 \%)$ & $28(28.0 \%)$ & \\
\hline T4 & $5(3.9 \%)$ & $3(60.0 \%)$ & $2(40.0 \%)$ & \\
\hline Nodal status & & & & 0.520 \\
\hline No & 31 (24.0\%) & $22(71.0 \%)$ & $9(29.0 \%)$ & \\
\hline N1 & $98(76.0 \%)$ & $71(72.4 \%)$ & 27 (27.6\%) & \\
\hline State of metastasis & & & & 0.310 \\
\hline MO & 125 (96.9\%) & $91(72.8 \%)$ & $34(27.2 \%)$ & \\
\hline M1 & $4(3.1 \%)$ & $2(50.0 \%)$ & $2(50.0 \%)$ & \\
\hline Grade & & & & 0.001 \\
\hline G1 & $8(6.2 \%)$ & $6(75.0 \%)$ & $2(25.0 \%)$ & \\
\hline $\mathrm{G} 2$ & $64(49.6 \%)$ & 55 (85.9\%) & $9(14.1 \%)$ & \\
\hline G3 & 57 (44.2\%) & $32(56.1 \%)$ & 25 (43.9\%) & \\
\hline
\end{tabular}

( $\mathrm{p}=0.001$ and 0.003 , respectively). A borderline significance was observed for histological grade $(\mathrm{p}=0.058)$.

The Kaplan-Meier analysis (Figure 2E) of grouped Sirt1 expression (IRS $\leq 6,>6$ ) was highly prognostic of poor overall survival for those patients with high Sirt1 expression with a mean postsurgical survival of 13.0 vs. 54.1 months (log-rank test: $\mathrm{p}=0.002)$.

\section{Multivariate survival analysis}

In multivariate Cox regression analysis (Table 3), high Sirt1 expression was significantly related to shorter overall survival (HR 1.647, 95\%CI 1.010-2.687, $\mathrm{p}=0.045$ ), independently of the degree of histological differentiation and WHO stage.

\section{Cellular effects of Sirt1 overexpression}

To test whether high Sirt1 expression also has a cellular effect in vitro, we performed overexpression experiments in both cell lines, MiaPaCa-2 and PANC-1, respectively, using 


\section{MiaPaCa-2 PANC-1}

\section{Sirt1} Actin
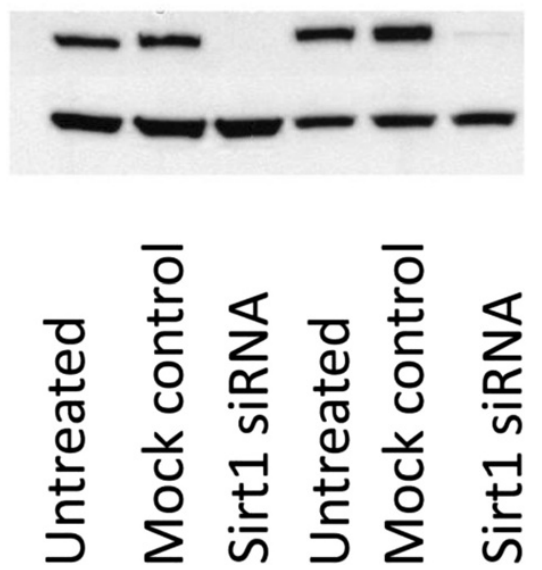

Figure 1 Immunblots with the antibody against Sirt1. While endogenous Sirt1 levels were detected by the antibody, knockdown of Sirt1 by siRNA in MiaPaCa-2 and PANC-1 cells led to a complete abrogation of the immunosignal indicating that the antibody binds specifically to its target protein.

flag-tagged Sirt1. Overexpression of GFP served as control. Figure 3A) shows immunoblots for endogenous and overexpressed Sirt1 in both cell lines. Cells overexpressing Sirt1 showed a markedly stronger immunosignal compared to their untransfected counterparts, which can also be depicted quantitatively as displayed in Figure 3B). Compared to GFP transfected cells, both cell lines showed statistically significantly increased amounts of viable, proliferating cells upon transfection with flag-tagged Sirt1 as determined by MTT assay (Figure 4) and Xcelligence proliferation assays (data not shown).

\section{Nicotinamide and gefitinib treatment in cells with endogenous or overexpressed Sirt1}

Inhibition of Sirt1 by increasing concentrations of nicotinamide led to a stepwise decrease of viable cells as depicted in Figure 5. Gefitinib treatment with concentrations of $50 \mu \mathrm{M}$ showed similar effects as observed for the application of $25 \mathrm{mM}$ nicotinamide. Interestingly, combinatory treatment with $50 \mu \mathrm{M}$ gefitinib and $25 \mathrm{mM}$ or $40 \mathrm{mM}$ nicotinamide showed a synergistic effect on cell viability, which was observed in both cell lines.

Next, we asked whether inhibition of Sirt 1 by nicotinamide may counterbalance the beneficial effect on cell survival triggered by Sirt1 overexpression. We found that application of $10 \mathrm{mM}$ and lower concentrations of nicotinamide, which in untransfected cells already showed a strong decrease of viable cell fractions compared to controls did not influence cell viability in cells overexpressing Sirt1, while higher concentrations of nicotinamide (Figure 6) abrogated increased cell viability mediated by overexpressed Sirt1.

\section{Cellular effects of cambinol, gemcitabine and gefitinib treatment \\ Proliferation assay}

Real time proliferation assays revealed an inhibition of cell growth of Mia-PaCa- 2 cells and PANC- 1 cells over a time period of 72 hrs upon treatment with cambinol. While for Mia-PaCa-2 comparably lower concentrations of cambinol (25 and $50 \mu \mathrm{M}$ ) were necessary to achieve this effect, for PANC-1 cells concentrations up to $100 \mu \mathrm{M}$ had to be
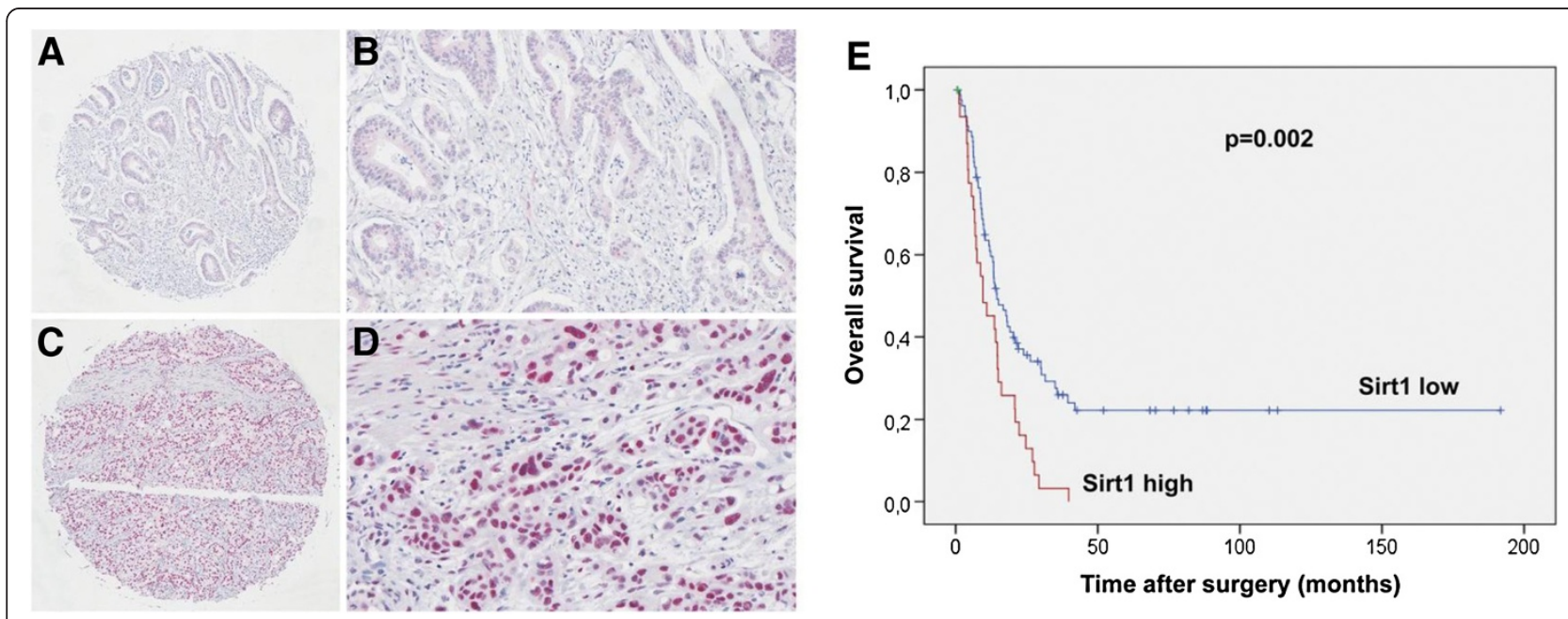

Figure 2 Sirt1 expression in PDAC. A) (overview, x5) and B (x20) show nuclear Sirt localization with a weak staining signal. C) (overview, x5) and $\mathbf{D}(x 20)$ display a strong nuclear Sirt1 immunosignal. E) Kaplan-Meier curve for postsurgical survival according to Sirt1 expression. The given p-value was calculated in a log-rank test. The red line indicates tumors of patients with high Sirt1 expression whereas the blue line indicates those with low Sirt1 expression. 
Table 2 Univariate survival analysis for Sirt1 expression and clinico-pathological parameters in PDAC

\begin{tabular}{|c|c|c|c|c|c|c|}
\hline & & Cases & Events & Mean survival (months) & Standard error & Log-rank-test (p-value) \\
\hline \multirow[t]{2}{*}{ Age at diagnosis } & $<=65$ years & 56 & 42 & 50.1 & 10.5 & 0.564 \\
\hline & $>65$ years & 57 & 47 & 25.1 & 4.4 & \\
\hline \multirow[t]{5}{*}{ WHO stage } & I & 11 & 8 & 29.4 & 9.8 & 0.001 \\
\hline & $\| \mathrm{A}$ & 17 & 10 & 52.8 & 11.9 & \\
\hline & $\| B$ & 78 & 64 & 33.7 & 7.0 & \\
\hline & III & 3 & 3 & 5.6 & 0.8 & \\
\hline & IV & 4 & 4 & 11.9 & 4.1 & \\
\hline \multirow[t]{4}{*}{ Tumor stage } & $\mathrm{T} 1$ & / & / & / & / & \\
\hline & $\mathrm{T} 2$ & 22 & 17 & 29.6 & 6.8 & 0.003 \\
\hline & T3 & 88 & 69 & 39.6 & 7.3 & \\
\hline & T4 & 3 & 3 & 5.6 & 0.8 & \\
\hline \multirow[t]{2}{*}{ Nodal status } & No & 30 & 20 & 43.3 & 8.8 & 0.060 \\
\hline & N1 & 83 & 69 & 32.2 & 6.6 & \\
\hline \multirow[t]{2}{*}{ State of metastasis } & MO & 109 & 85 & 42.4 & 6.8 & 0.268 \\
\hline & M1 & 4 & 4 & 11.9 & 4.1 & \\
\hline \multirow[t]{3}{*}{ Grade } & G1 & 7 & 6 & 16.8 & 2.5 & 0.058 \\
\hline & G2 & 54 & 39 & 37.3 & 6.0 & \\
\hline & G3 & 52 & 44 & 29.7 & 7.9 & \\
\hline \multirow[t]{2}{*}{ SIRT1-expression } & low & 81 & 58 & 54.1 & 8.9 & 0.002 \\
\hline & high & 32 & 31 & 13.0 & 1.7 & \\
\hline
\end{tabular}

applied (Figure 7). Combination of cambinol and gefitinib led to a synergistic inhibitory effect on cell growth for both cell lines. As in the previous experiment slightly higher concentrations for cambinol as well as for gefitinib were used to achieve comparable results in PANC-1 cells.

As expected in Mia-PaCa-2 comparably low concentrations of gemcitabine alone led to strong growth inhibitory

Table 3 Multivariate survival analysis (Cox regression model) including tumor stage and grade in PDAC

\begin{tabular}{llll}
\hline \multicolumn{4}{l}{ Overall survival } \\
\hline WHO stage & PR & 95\%Cl & p-value \\
I & 1 & & \\
IIA & 0.57 & $0.22-1.45$ & \\
IIB & 1.11 & $0.53-2.24$ & 0.029 \\
III & 4.92 & $1.20-20.16$ & \\
IV & 1.88 & $0.56-6.33$ & 0.298 \\
Grade & & & \\
G1/G2 & 1 & & 0.045 \\
G3 & 1.28 & $0.81-2.03$ & \\
SIRT1-expression & & & \\
low & 1 & $1.01-2.69$ & \\
high & 1.65 & & \\
\hline
\end{tabular}

effects, while in PANC-1 comparably higher concentrations were necessary (data not shown). Although we tested a multitude of different treatment schemes, a synergistic effect for treatment with gemcitabine and cambinol in combination was not observed (data not shown).

\section{Cell cycle analysis}

To determine the nature of the cellular growth inhibition, we performed FACS analyses. For PANC-1 cells treated with either cambinol or gefitinib alone or in combination, a sub-G1 peak was observed indicating apoptosis (Figure 8A), which was also evident by demonstrating cleaved PARP by immunoblot (Figure 8B). Cell cycle analysis of Mia-Paca-2 cells showed a cell cycle arrest for different concentrations of cambinol (25 and $50 \mu \mathrm{M})$ and for a combinatory regimen of cambinol and gefitinib (Additional file 1: Figure S1), but in our experimental setting no apparent apoptosis induction.

\section{Senescence analysis}

Upon treatment with cambinol, we observed for both cell lines a population of growth-arrested cells with a flattened, elongated appearance and extended cellular protrusions (Additional file 2: Figure S2A). As exemplified in Additional file 2: Figure S2B, immunblotting revealed a marked upregulation of $\mathrm{y}-\mathrm{H} 2 \mathrm{AX}$ in Mia-Paca-2 cells indicating a senescent phenotype. 


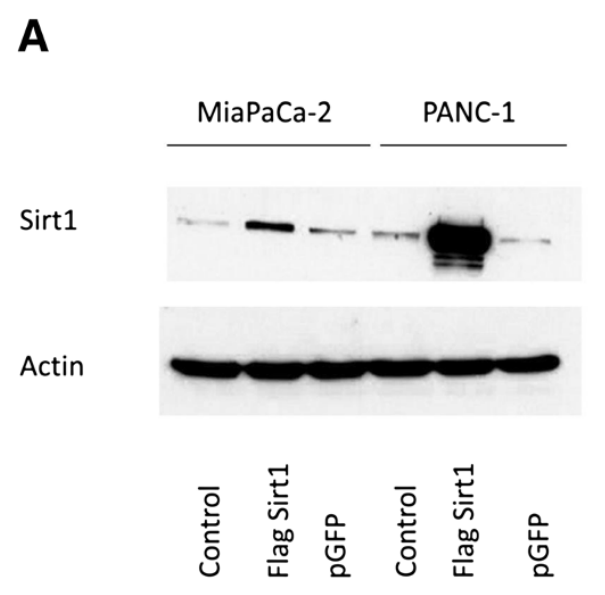

\section{B Densitometry of Immunoblots}

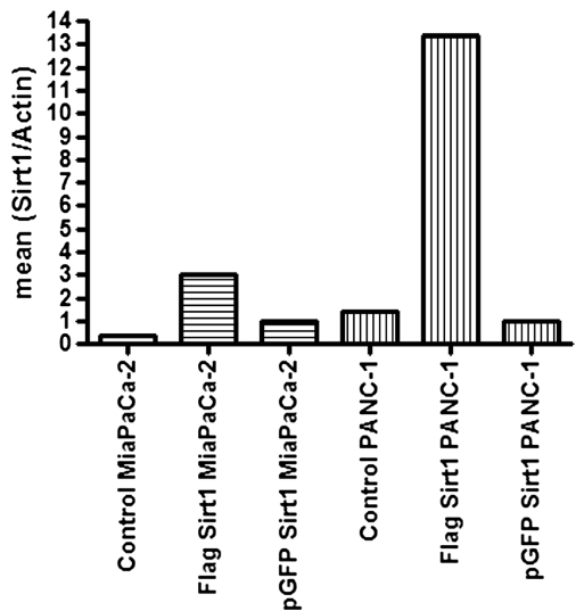

Figure 3 Immunblots with the antibody against Sirt1 for MiaPaCa-2 and PANC-1. A) Endogenous protein levels showed a comparably weaker immunosignal than cells overexpressing Sirt1. B) The blots were scanned and analysed quantitatively using ImageJ. The values were normalized for pGFP. The graphs show strongly increased immunosignal densities for cells that overexpress Sirt1.

High concentrations of cambinol lead to abrogation of Sirt1 Immunoblotting of cells treated with cambinol 100 or $200 \mu \mathrm{M}$ revealed an extinction of the Sirt1 protein as compared to controls treated with DMSO only (Figure 9). While this effect was repeatedly observed in Mia-Paca-2 cells after $24 \mathrm{hrs}, 48 \mathrm{hrs}$ and $72 \mathrm{hrs}$ of cambinol treatment, for PANC-1 cells only high concentrations of cambinol applied for 72 hrs led to a similar effect.

\section{Discussion}

This is the first study that demonstrates Sirt1 to be an independent prognosticator in PDAC with high Sirt1 expression indicating poor outcome. Moreover, our data argue for a functional role of Sirt 1 during tumorigenesis indicating that Sirt1 is not only a biomarker but a potentially oncogenic protein in the PDAC context, whose overexpression leads to increased cell viability in both cell lines, while pharmacological inhibition leads to a concentration-dependent stepwise decrease of viable cells. Cambinol treatment negatively interferes with cell cycle progression (in MiaPaCa-2 cells) and induces apoptosis (in PANC-1 cells) as well as senescence (both cell lines). These observations are in line with Wauters et al. [33] showing an enhancing effect for cell viability and regulatory function of Sirt1 for acinar-to-ductal metaplasia in pancreatic carcinogenesis. The latter results also match data presented by Zhao et al. [28] who reported that utilizing small hairpin RNA Sirt1 knockdown led to increased apoptosis and senescence in PANC-1 cells. However, we failed to observe a synergistic effect of Sirt1 inhibition with Gemcitabine treatment as reported by Zhao et al. [28]. This divergent result may be attributed to the distinct targeting approach in our study, which uses cambinol, a clinically applicable drug with promising anti-cancer effects in animal models of skin cancer and Burkitt's lymphoma as well as in several cancer cell lines [34].
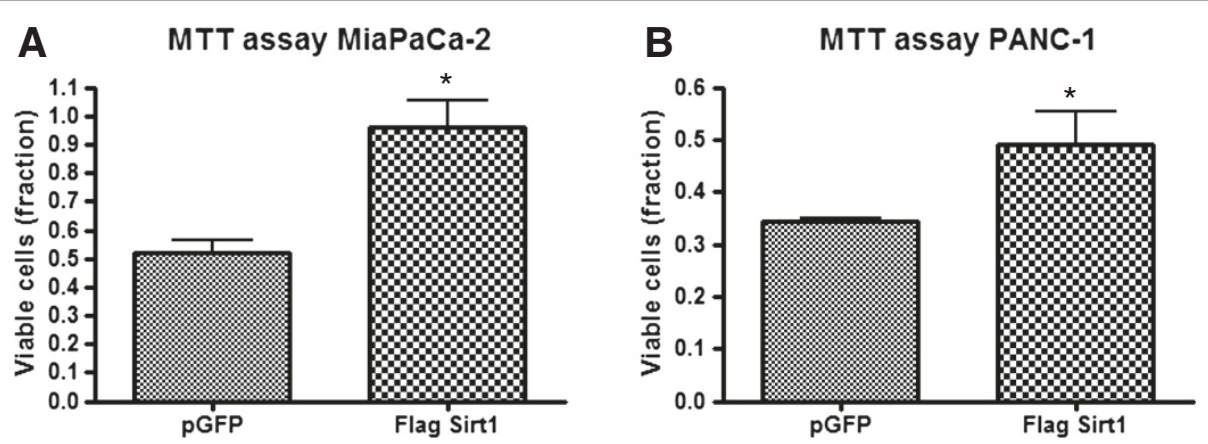

Figure 4 Cell viability of MiaPaCa-2 and PANC-1 cells assessed by MTT test after treatment with flag-tagged Sirt1 and GFP, respectively. A) MiaPaCa-2, B) Panc-1. The test was carried out 3 days after transfection. Bars represent average \pm standard deviation (SD) of three independent experiments. ${ }^{*} \mathrm{P}<0.05$. 


\section{A}

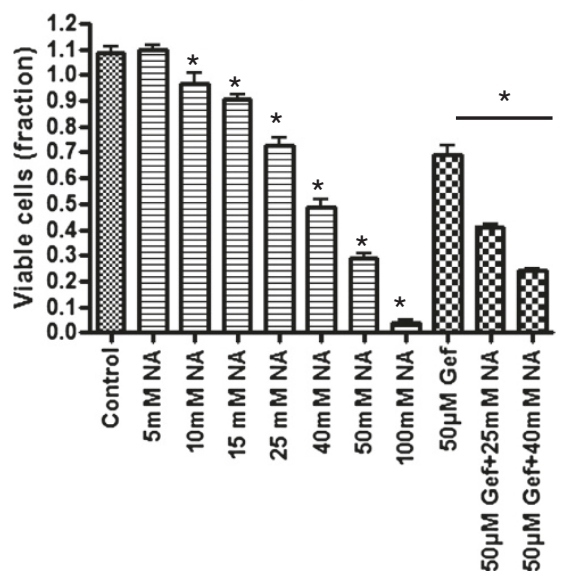

B

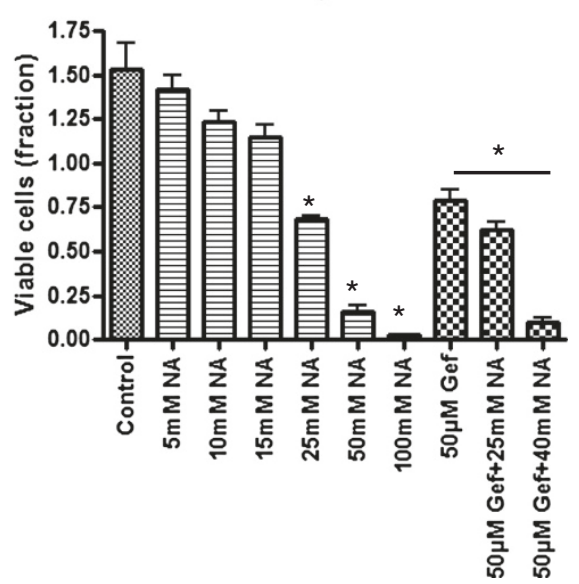

Figure 5 Cell viability of MiaPaCa-2 and PANC-1 cells assessed by MTT test after treatment with nicotinamide (NA) and gefitinib (Gef), respectively. A) MiaPaCa2 cells, B) PANC-1 cells. Concentrations were used as indicated. Bars represent average \pm standard deviation (SD) of three independent experiments. ${ }^{*} P<0.05$.

Interestingly, we detected an application time- and concentration-dependent loss of Sirt1 protein upon cambinol treatment. The underlying cause for this effect, which abrogates Sirt1-function, remains to be elucidated and may be due to protein degradation.

Consistent with the results by Zhao et al. [28] obtained by immunhistochemistry, qPCR and western blotting, we observed a variable expression of Sirt1 in PDACs but did not see a positive correlation of Sirt1 expression with age, tumor size, and lymphatic spread. The different findings may be explained by distinct cohort characteristics including cohort size, age, and sex. However and in contrast to Zhao et al., we observed a strong correlation with higher tumor grades, i.e. the less differentiated the cancer cells are the more Sirt1 expression they exhibit. This finding is of interest since there are reports that implicate Sirt1 in the regulation of cellular differentiation and dedifferentiation processes [35,36]. Dedifferentiation and the associated phenomenon of epithelial-to-mesenchymal-transition play an essential role in the development of early local and distant tumor spread. Observations that link high Sirt1 expression to poorly differentiated cancers were also made by other investigators for hepatocellular carcinoma [37], prostate cancer [38] and glioblastoma [39].

The association between high Sirt1 expression and poor histological grade may also explain why in our cohort Sirt1
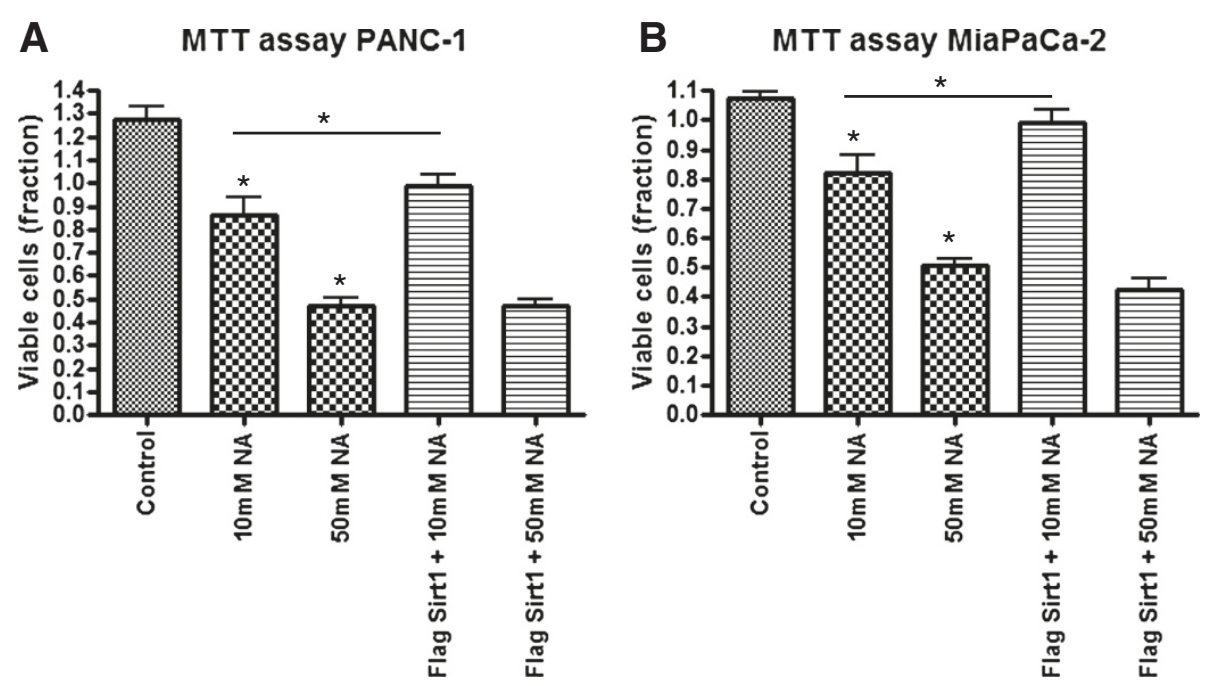

Figure 6 Cell viability of MiaPaCa-2 and PANC-1 cells assessed by MTT test after treatment with flag-tagged Sirt1 and/or nicotinamide (NA) respectively. A) PANC-1 cells, B) MiaPaCa-2 cells. Concentrations were used as indicated. Bars represent average \pm standard deviation (SD) of three independent experiments. ${ }^{*} \mathrm{P}<0.05$. 

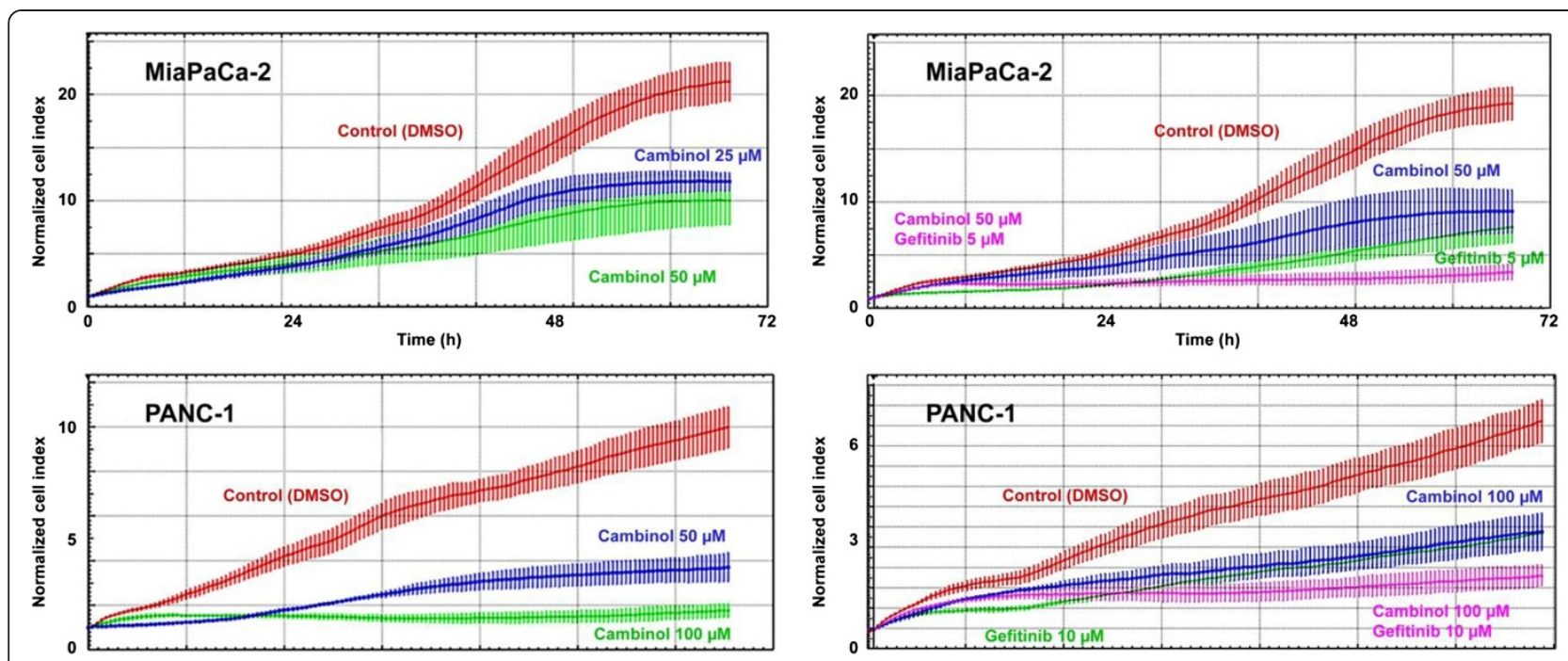

Figure 7 Real-time cell proliferation assays (X-Celligence system). Dynamic cell proliferation of MiaPaCa-2 and PANC-1 cells plated on the EPlates 96 was monitored at 30-min intervals from the time of plating until the end of the experiment.

expression is associated with poor outcome regardless of the tumor stage as shown by its prognostic independency in multivariate survival analysis. A Sirt1 positive and poorly differentiated tumor may have acquired a biological profile that allows for e.g. early systemic spread of -clinically undetectable- micrometastases in lymph nodes and distant organs leading to impaired survival regardless of the tumor size and metastases detected at the point of initial tumor diagnosis. A recent study by Nalls and colleagues [40] showed that SAHA-induced micro-RNA 34a (miR34a) expression in human pancreatic cancer cells putatively directly inhibited Sirt1 expression by binding within the 3'UTR of Sirt1. On cellular level, restoration of miR34a expression led to growth inhibition as well as decreased epithelial to mesenchymal transition (EMT) and invasion. Although miR34a does not exclusively target Sirt1, this recent study further argues for an oncogenic role of Sirt1 in PDAC development. Recent results obtained by Pramanik et al. corroborate this view [41].

Functional studies indicate that the subcellular localization of Sirt1 might have functional implications in carcinogenesis. Wauters et al. [33] recently provided evidence that there is nuclear to cytoplasmic shuttling of Sirt1 in rat and mouse acinar cells with potential tumorigenic implications in the acinar to ductal metaplasia carcinogenesis model of PDAC. They also reported on cytoplasmic localization of Sirt1 in exocrine cells of the human pancreas. However, investigating human tissue samples of fully developed pancreatic ductal adenocarcinoma, we only detected nuclear localized Sirt1. This may have several reasons. One potential explanation might be that endogenous cytoplasmic Sirt1 levels in comparison to nuclear expression levels are too low to be detected by our antibody. Another explanation would be that cytoplasmic
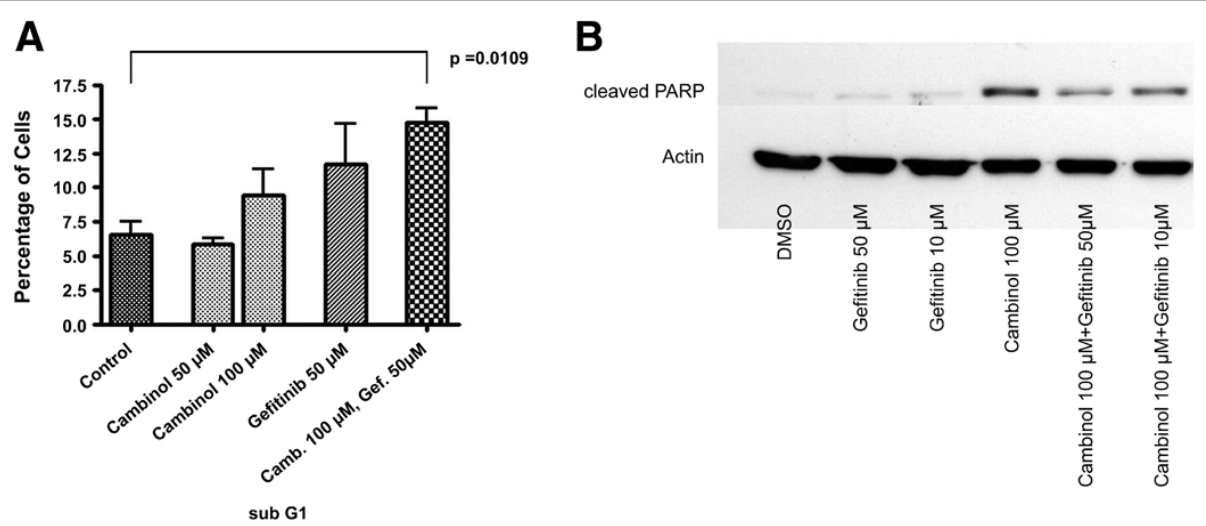

Figure 8 Cell cycle analysis and apoptosis in PANC-1 cells. A) Particularly combinatory therapy with gefitinib and cambinol led to a marked sub-G1 peak indicating apoptotic cells. B) Immunoblotting for cleaved PARP in PANC-1 cells. Reagents and concentrations as indicated. 


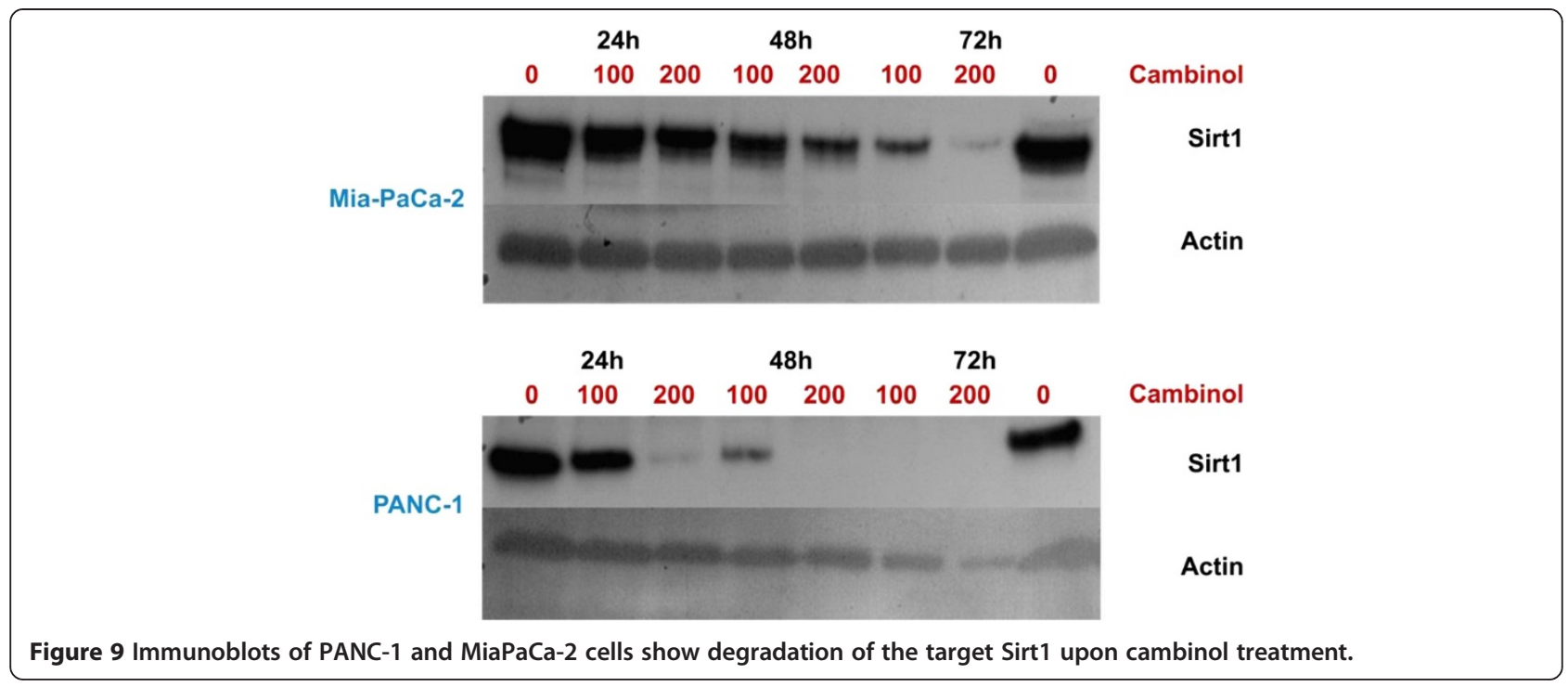

Sirt1 plays a major role in the development of carcinogenic precursors and nuclear Sirt1 has its place in the fully developed cancer. However, this has to be investigated in future functional studies.

Interestingly, following up the seminal work by Luo et al. and Vasiri et al. [6,7], a very recent study by $\mathrm{Li}$ and coworkers [42] explored the Sirt1-p53 axis in chronic myeloid leukemia (CML) and found that targeting of Sirt1 by either shRNA or the small molecule inhibitor tenovin-6 resulted in increased levels of acetylated p53 in CML CD34+ cells accompanied by increased transcriptional activity of p53. Abrogation of Sirt1 led to growth inhibition and reduced engraftment of the tumor cells. These effects were even more pronounced when cells were synergistically treated with the tyrosine kinase inhibitor imatinib. These data strengthen the view of a context-dependent tumorigenic impact of Sirt1 as also suggested by our results. Since p53 aberrations are commonly involved in PDAC tumorigenesis [43,44], it is tempting to speculate whether Sirt1 inhibition may help to restore the remaining functionally intact p53 pool. Indeed, recent data [45] indicate that downregulation of Sirt1 by restoration of HIC1 (hypermethylated in cancer 1) leads to increased levels of acetylated p53 and upregulated p21 in pancreatic cancer. On cellular level, overexpressed HIC1, which in turn led to downregulation Sirt1 resulted in cell cycle arrest and apoptosis. Loss of p53 function has also been implicated in resistance to EGFR-targeting strategies [46], the latter having a limited but significant role in the treatment of PDACs [47]. Interestingly, we observed a synergistic impact of combined Sirt1- and EGFR-inhibition suggesting a functional interdependence in PDACs, whose molecular details remain to be explored. In prostatic cancer cells Byles and colleagues [48] observed Sirt1 to modulate EMT upon EGF signalling via the induction of the transcription factor
ZEB1. Although it remains to be investigated whether this mechanism works in PDACs, our data and these results may additionally point to a therapeutic rationale for combined EGFR/Sirt1 inhibition.

While a number of small molecule inhibitors of class I and II HDACs are currently in clinical trials for the treatment of malignancies of various organ origins [49], SIRT1 inhibition is currently only investigated in a phase I trial of patients with Huntington's disease.

\section{Conclusions}

In conclusion, there is accumulating evidence that Sirt1 has an oncogenic role in PDACs and provided that further studies are able to reproduce and extent the data presented herein towards mouse model systems, a clinical trial for patients with PDAC, whose outcome and treatment options are extremely limited for the vast majority of patients, may be worthwhile to consider.

\section{Additional files}

Additional file 1: Figure S1. Cell cycle analysis of MiaPaCa-2 cells showing growth arrest of tumor cells upon treatment as indicated.

Additional file 2: Figure S2. A) PANC-1 and MiaPaCa-2 cells show a flattened phenotype with cellular protrusions. B) Immunoblots of MiaPaCa-2 cells treated with cambinol and gefitinib as indicated showed upregulation of $\mathrm{y}-\mathrm{H} 2 \mathrm{AX}$.

Competing interest

The authors indicate no potential conflicts of interest.

\section{Authors' contributions}

AS and WW designed the study, supervised research, analyzed the data, and wrote the paper. AS, VE, and KL performed experiments and analyzed data. $V$ Ehemann performed cell cycle experiments. FK, BS, AW, BG, CK, MB and PN provided patient samples, characterized some of the samples, collected data and assisted in writing the paper. All authors read and approved the final manuscript. 


\section{Author details}

${ }^{1}$ Institute of Pathology, and National Center for Tumor Diseases (NCT) University Hospital Heidelberg, Heidelberg, Germany. ${ }^{2}$ Institute of Pathology, Charité-Universitätsmedizin, Berlin, Germany. ${ }^{3}$ Department of General, Visceral and Transplantation Surgery, Charité-Universitätsmedizin, Berlin, Germany.

Received: 25 April 2013 Accepted: 25 September 2013

Published: 2 October 2013

\section{References}

1. Hong SM, Park JY, Hruban RH, Goggins M: Molecular signatures of pancreatic cancer. Arch Pathol Lab Med 2011, 135:716-27.

2. Hidalgo M: Pancreatic cancer. N Engl J Med 2010, 362:1605-1617.

3. Li D, Xie K, Wolff R, Abbruzzese JL: Pancreatic cancer. Lancet 2004, 363:1049-1057.

4. Vincent A, Herman J, Schulick R, Hruban RH, Goggins M: Pancreatic cancer. Lancet 2011, 378:607-20

5. Singh P, Srinivasan R, Wig JD: Major molecular markers in pancreatic ductal adenocarcinoma and their roles in screening, diagnosis, prognosis, and treatment. Pancreas 2011, 40:644-652.

6. Vaziri H, Dessain K, Ng Eaton E, Imai SI, Frye RA, Pandita TK, Guarente L, Weinberg RA: hSIR2(SIRT1) Functions as an NAD-Dependent p53 Deacetylase. Cell 2001, 107:149-159.

7. Luo J, Nikolaev AY, Imai S, Chen D, Su F, Shiloh A, Guarente L, Gu W: Negative Control of p53 by Sir2alpha Promotes Cell Survival under Stress. Cell 2001, 107:137-148.

8. Jeong J, Juhn K, Lee H, Kim SH, Min BH, Lee KM, Cho M, Park GH, Lee KH: SIRT1 Promotes DNA Repair Activity and Deacetylation of Ku70. Exp Mol Med 2007, 39:8-13.

9. Ikenoue T, Inoki K, Zhao B, Guan KL: PTEN Acetylation Modulates Its Interaction with PDZ Domain. Cancer Res 2008, 68:6908-6912.

10. Dai JM, Wang ZY, Sun DC, Lin RX, Wang SQ: SIRT1 Interacts with p73 and Suppresses p73-Dependent Transcriptional Activity. J Cell Physiol 2007, 210:161-166.

11. Yeung F, Hoberg JE, Ramsey CS, Keller MD, Jones DR, Frye RA, Mayo MW: Modulation of NF-kappaB-Dependent Transcription and Cell Survival by the SIRT1 Deacetylase. EMBO J 2004, 23:2369-2380.

12. Motta MC, Divecha N, Lemieux M, Kamel C, Chen D, Gu W, Bultsma Y, McBurney M, Guarente L: Mammalian SIRT1 Represses Forkhead Transcription Factors. Cell 2004, 116:551-563.

13. Guarani V, Deflorian G, Franco CA, Kruger M, Phng LK, Bentley K, Toussaint L, Dequiedt F, Mostoslavsky R, Schmidt MH: Acetylation-Dependent Regulation of Endothelial Notch Signalling by the SIRT1 Deacetylase. Nature 2011, 473:234-238.

14. Lim JH, Lee YM, Chun YS, Chen J, Kim JE, Park JW: Sirtuin 1 Modulates Cellular Responses to Hypoxia by Deacetylating Hypoxia-Inducible Factor 1alpha. Mol Cell 2010, 38:864-878.

15. Dioum EM, Chen R, Alexander MS, Zhang Q, Hogg RT, Gerard RD, Garcia JA: Regulation of Hypoxia-Inducible Factor 2alpha Signaling by the StressResponsive Deacetylase Sirtuin 1. Science 2009, 324:1289-1293.

16. Firestein R, Blander G, Michan S, Oberdoerffer P, Ogino S, Campbell J, Bhimavarapu A, Luikenhuis S, de Cabo R, Fuchs C, Hahn WC, Guarente LP Sinclair DA: The SIRT1 Deacetylase Suppresses Intestinal Tumorigenesis and Colon Cancer Growth. PLoS One 2008, 3:e2020.

17. Fan W, Luo J: Regulates UV-Induced DNA Repair through Deacetylating XPA. Mol Cell 2010, 39:247-258

18. Kume S, Haneda M, Kanasaki K, Sugimoto T, Araki S, Isshiki K, Isono M, UzU T, Guarente L, Kashiwagi A, Koya D: SIRT1 Inhibits Transforming Growth Factor Beta-Induced Apoptosis in Glomerular Mesangial Cells via Smad7 Deacetylation. J Biol Chem 2007, 282:151-158

19. Zhang Y, Zhang M, Dong H, Yong S, Li X, Olashaw N, Kruk PA, Cheng JQ, Bai W, Chen J: Deacetylation of Cortactin by SIRT1 Promotes Cell Migration. Oncogene 2009, 28:445-460.

20. Tissenbaum HA, Guarente L: Increased dosage of a sir-2 gene extends lifespan in Caenorhabditis elegans. Nature 2001, 410:227-230.

21. Viswanathan M, Guarente L: Regulation of Caenorhabditis elegans lifespan by sir-2.1 transgenes. Nature 2011, 477:E1-2.

22. Rogina B, Helfand SL: Sir2 mediates longevity in the fly through a pathway related to calorie restriction. Proc Natl Acad Sci U S A 2004 101:15998-6003.
23. Burnett $C$, Valentini $S$, Cabreiro F, Goss M, Somogyvári M, Piper MD, Hoddinott M, Sutphin GL, Leko V, McElwee JJ, Vazquez-Manrique RP, Orfila AM, Ackerman D, Au C, Vinti G, Riesen M, Howard K, Neri C, Bedalov A, Kaeberlein M, Soti C, Partridge L, Gems D: Absence of effects of Sir2 overexpression on lifespan in C. elegans and Drosophila. Nature 2011, 477:482-485.

24. Brooks CL, Gu W: How does SIRT1 affect metabolism, senescence and cancer? Nat Rev Cancer 2009, 9:123-128.

25. Marshall GM, Liu PY, Gherardi S, Scarlett CJ, Bedalov A, Xu N, Iraci N, Valli E, Ling D, Thomas W, van Bekkum M, Sekyere E, Jankowski K, Trahair T, Mackenzie KL, Haber M, Norris MD, Biankin AV, Perini G, Liu T: SIRT1 Promotes N-Myc Oncogenesis through a Positive Feedback Loop Involving the Effects of MKP3 and ERK on N-Myc Protein Stability. PLOS Genet 2011, 7:e1002135.

26. Serrano M: SIRT1 Transgenic and Cancer Models. Orlando, FL: Proceedings of the 102nd Annual Meeting of the AACR; 2011. Presentation SY11-03.

27. Saunders $L R$, Verdin E: Sirtuins: critical regulators at the crossroads between cancer and aging. Oncogene 2007, 26:5489-5504

28. Zhao G, Cui J, Zhang JG, Qin Q, Chen Q, Yin T, Deng SC, Liu Y, Liu L, Wang $B$, Tian K, Wang GB, Wang CY: SIRT1 RNAi knockdown induces apoptosis and senescence, inhibits invasion and enhances chemosensitivity in pancreatic cancer cells. Gene Ther 2011, 18:920-928.

29. Sobin LH, Gospodarowicz MK, Wittekind C: UICC: TNM classification of malignant tumors. 7th edition. Oxford: Wiley-Blackwell; 2009.

30. Bosman FT, Carneiro F, Hruban RH, Theise ND (Eds): WHO Classification of Tumours of the Digestive System (IARC WHO Classification of Tumours), The International Agency for Research on Cancer (Author). 4th edition. ; 2010.

31. Brunet A, Sweeney LB, Sturgill JF, Chua KF, Greer PL, Lin Y, Tran H, Ross SE, Mostoslavsky R, Cohen HY, Hu LS, Cheng HL, Jedrychowski MP, Gygi SP, Sinclair DA, Alt FW, Greenberg ME: Stress-dependent regulation of FOXO transcription factors by the SIRT1 deacetylase. Science 2004, 303:2011-2015.

32. Ehemann V, Hashemi B, Lange A, Otto HF: Flow cytometric DNA analysis and chromosomal aberrations in malignant glioblastomas. Cancer Lett 1999, 138:101-106.

33. Wauters E, Sanchez-Arévalo Lobo VJ, Pinho AV, Mawson A, Herranz D, Wu J, Cowley MJ, Colvin EK, Njicop EN, Sutherland RL, Liu T, Serrano M, Bouwens L, Real FX Biankin AV, Rooman I: Sirtuin-1 Regulates Acinar-to-Ductal Metaplasia and Supports Cancer Cell Viability in Pancreatic Cancer. Cancer Res 2013, 66:2357-2367.

34. Heltweg B, Gatbonton T, Schuler AD, Posakony J, Li H, Goehle S, Kollipara R, Depinho RA, Gu Y, Simon JA, Bedalov A: Antitumor activity of a smallmolecule inhibitor of human silent information regulator 2 enzymes. Cancer Res 2006, 66:4368-4377.

35. Simic P, Zainabadi K, Bell E, Sykes DB, Saez B, Lotinun S, Baron R, Scadden D, Schipani $E$, Guarente L: SIRT1 regulates differentiation of mesenchymal stem cells by deacetylating $\beta$-catenin. EMBO Mol Med 2013, 5:430-40.

36. Hong EH, Yun HS, Kim J, Um HD, Lee KH, Kang CM, Lee SJ, Chun JS, Hwang SG: Nicotinamide phosphoribosyltransferase is essential for interleukin1 beta-mediated dedifferentiation of articular chondrocytes via SIRT1 and extracellular signal-regulated kinase (ERK) complex signaling. J Biol Chem 2011, 286:28619-31.

37. Chen HC, Jeng YM, Yuan RH, Hsu HC, Chen YL: SIRT1 promotes tumorigenesis and resistance to chemotherapy in hepatocellular carcinoma and its expression predicts poor prognosis. Ann Surg Oncol 2012, 19:2011-9.

38. Huffman DM, Grizzle WE, Bamman MM, Kim JS, Eltoum IA, Elgavish A, Nagy TR: SIRT1 is significantly elevated in mouse and human prostate cancer Cancer Res 2007, 67:6612-8

39. Liu G, Yuan X, Zeng Z, Tunici P, Ng H, Abdulkadir IR, Lu L, Irvin D, Black KL, Yu JS: Analysis of gene expression and chemoresistance of CD133+ cancer stem cells in glioblastoma. Mol Cancer 2006, 5:67.

40. Nalls D, Tang SN, Rodova M, Srivastava RK, Shankar S: Targeting epigenetic regulation of miR-34a for treatment of pancreatic cancer by inhibition of pancreatic cancer stem cells. PLoS One 2011, 6e:24099.

41. Pramanik D, Campbell NR, Karikari C, Chivukula R, Kent OA, Mendell JT, Maitra A: Restitution of tumor suppressor microRNAs using a systemic nanovector inhibits pancreatic cancer growth in mice. Mol Cancer Ther 2011, 10:1470-1480.

42. Li L, Wang L, Li L, Wang Z, Ho Y, McDonald T, Holyoake TL, Chen W, Bhatia R: Activation of p53 by SIRT1 Inhibition Enhances Elimination of CML Leukemia Stem Cells in Combination with Imatinib. Cancer Cell 2012, 21:266-81. 
43. Barton CM, Staddon SL, Hughes CM, Hall PA, O'Sullivan C, Klöppel G, Theis B, Russell RC, Neoptolemos J, Williamson RC, et al: Abnormalities of the p53 tumour suppressor gene in human pancreatic cancer. $\mathrm{Br} J$ Cancer 1991, 64:1076-1082.

44. Ruggeri B, Zhang SY, Caamano J, DiRado M, Flynn SD, Klein-Szanto AJ: Human pancreatic carcinomas and cell lines reveal frequent and multiple alterations in the $\mathrm{p} 53$ and $\mathrm{Rb}-1$ tumor-suppressor genes. Oncogene 1992, 7:1503-1511.

45. Zhao G, Qin Q, Zhang J, Liu Y, Deng S, Liu L, Wang B, Tian K, Wang C: Hypermethylation of HIC1 Promoter and Aberrant Expression of HIC1/SIRT1 Might Contribute to the Carcinogenesis of Pancreatic Cancer. Ann Surg Oncol 2012. doi:DOI 10.1245/s10434-012-2364-9. May 3. [Epub ahead of print].

46. Huang S, Benavente S, Armstrong EA, Li C, Wheeler DL, Harari PM: p53 modulates acquired resistance to EGFR inhibitors and radiation. Cancer Res 2011, 71:7071-7079.

47. Lowery MA, O'Reilly EM: Genomics and pharmacogenomics of pancreatic adenocarcinoma. Pharmacogenomics J 2012, 12:1-9.

48. Byles V, Zhu L, Lovaas JD, Chmilewski LK, Wang J, Faller DV, Dai Y: SIRT1 induces EMT by cooperating with EMT transcription factors and enhances prostate cancer cell migration and metastasis. Oncogene 2012, 31:4619-29.

49. Marks PA, Breslow R: Dimethyl sulfoxide to vorinostat: development of this histone deacetylase inhibitor as an anticancer drug. Nat Biotechnol 2007, 25:84-90.

doi:10.1186/1471-2407-13-450

Cite this article as: Stenzinger et al: High SIRT1 expression is a negative prognosticator in pancreatic ductal adenocarcinoma. BMC Cancer 2013 13:450.

\section{Submit your next manuscript to BioMed Central and take full advantage of:}

- Convenient online submission

- Thorough peer review

- No space constraints or color figure charges

- Immediate publication on acceptance

- Inclusion in PubMed, CAS, Scopus and Google Scholar

- Research which is freely available for redistribution 